\title{
La influencia de los circuitos turísticos y las ferias rurales en el nivel socioeconómico de las familias urbanas y rurales del Valle del Mantaro 2010-2014
}

\section{The influence of tourist circuits and rural fairs on the socio-economic level of the urban and rural families of the Mantaro Valley 2010-2014}

Filoter Tello Y.' \& Mauro R. De la Cruz'

Facultad de Sociología de la Universidad Nacional del Centro del Perú

Email: ftelloya@hotmail.com

\section{RESUMEN}

El objetivo del trabajo fue identificar la influencia de los circuitos turísticos y las ferias rurales en el nivel socioeconómico de las familias urbanas y rurales beneficiarias de estas actividades en el Valle del Mantaro, durante los años 2010 al 2014, en el proceso de ejecución, se utilizaron los métodos descriptivos y analíticos de sus componentes con el enfoque sistémico, se arribó a las siguientes conclusiones, las ferias rurales y los circuitos turísticos si contribuyen en la mejora de las condiciones sociales de las familia como en la educación, salud, vivienda y viene generado fuente de trabajo y consecuentemente ha incrementado los niveles de ingreso económico para las familias que ofertan productos a través de las diversas feria rurales en el ámbito espacial del Valle del Mantaro, del mismo modo los circuitos turísticos contribuyen en la mejora de las condiciones socioeconómicas que ofertan productos turísticos, demostrando de esta manera que las ferias y los circuitos turísticos deben ser promocionados a través de los diversos medios de información. El Valle del Mantaro, uno de los valles más grandes de la sierra del Perú, es un lugar muy atractivo por la cercanía a la ciudad de Lima y por la riqueza de sus recursos turísticos tanto naturales y convencionales. En el valle del Mantaro, existen diversas zonas muy atractivas y aprovechable a través de los circuitos turísticos, en función a los atractivos turísticos como los complejos arqueológicos, los paisajes naturales y las fiestas patronales, los mismos que complementan con la gastronomía muy diversificada, con la música, danzas y los atuendos multicolores y forman parte de los paquetes turísticos y son aprovechados por la demanda y la necesidad de esparcimiento y de recreación de la población huancaína y de turistas nacionales y extranjeros, quienes necesitan recrearse y de este modo se observa que todos los días salen una flota de 5 a 8 carros a cargo del Yuri Tours y otras empresas.

\begin{abstract}
The objective of this work was to identify the influence of tourist circuits and rural fairs on the socioeconomic level of urban and rural families who benefited from these activities in the Mantaro Valley during the years 2010-2014. Descriptive and analytical methods of its components with the systemic approach, the following conclusions were reached: rural fairs and tourist circuits if they contribute to the improvement of the social conditions of the family, such as education, health, housing and source Of work and consequently has increased the levels of economic income for the families that offer products through the various rural fairgrounds in the Mantaro Valley space, in the same way the tourist circuits contribute in the improvement of the socioeconomic conditions that offer products Tourism, demonstrating in this way that fairs and tourist circuits should be promoted through the various media. The Mantaro Valley, one of the largest valleys in the Peruvian sierra, is a very attractive place because of the proximity to the city of Lima and the richness of its natural and conventional tourist resources. In the valley of the Mantaro, there are several very attractive areas and can be used through tourist circuits, depending on the tourist attractions such as archaeological complexes, natural landscapes and patron saint festivities, which complement the highly diversified gastronomy, with Music, dances and multicolored outfits and are part of the tourist packages and are taken advantage of by the demand and the need for recreation and recreation of the population of Huancaína and of national and foreign tourists, who need recreation and in this way it is observed that Every day a fleet of 5 to 8 cars comes out of Yuri Tours and other companies.
\end{abstract}

\section{Palabras clave | Key words:}

circuitos turísticos, feria rurales y nivel socioeconómico.

tourist circuits, rural fair and socioeconomic level. 


\section{INTRODUCCIÓN}

La motivación que permitió investigar, respondió a la necesidad de conocer la importancia de la promoción turística y su articulación con las ferias rurales que funcionan como redes socioeconómicos de gran importancia, y la influencia en el nivel socioeconómico y cambio de patrones culturales de las familias urbanas y rurales beneficiarias de estas actividades en el Valle del Mantaro y como objetivos específicos Analizar la influencia de los circuitos turísticos y las ferias rurales en el nivel de ingreso, en las condiciones sociales y en el cambio patrones culturales.

En el valle del Mantaro, existen varias zonas llamadas los circuitos turísticos, en función a los atractivos turísticos como los complejos arqueológicos, los paisajes naturales y las fiestas patronales que se complementan con la gastronomía diversa, con la música, danzas y los atuendos multicolores y forman parte de los paquetes turísticos y son aprovechados por la demanda de la necesidad de esparcimiento y de recreación de la población huancaína y de turistas nacionales y extranjeros, quienes necesitan recrearse y de este modo se observa que todos los días salen una flota de 5 a 8 carros a cargo del Yuri Tours. El primer circuito turístico comprende los lugares tradicionales de Hualhuas, san jerónimo, la planta lechera de concepción, la laguna de Paca, Ingenio, y terminan en el Convento de Santa Rosa de Ocopa de los padres franciscanos. El segundo circuito turístico, comprende la visita a Chupaca, Cani Cruz, a la capilla de Copón, el más antiguo del Perú, Arwaturo, laguna de Ñahuinpuquio, el complejo arqueológico de Warivilca y termina con la visita al manantial de la fidelidad. El tercer circuito es a la selva central y visitan al Señor de Murhuay, puente Kimiri, Pampamichi, catarata de Tirol, Mariposarios, procesados de café, gruta de Guagapo, terrazas y andenería de Tarmatambo, restos arqueológicos, el mirador natural, santuario del Señor de Murhuay.

El cuarto circuito aventura al nevado de Huaytapallana, incluye la visitan a la Virgen de las Nieves, caminata al nevado, visita a las lagunas de Yanaucsha, Wacracocha, chuspicocha y Lazo huntay.

El otro circuito es city tours Wanka que ofrece la visita al parque de la Constitución, Torre Torre, parque de la Identidad Wanka, Plaza Hua- manmarca. Para este propósito se cuenta con el mapa turístico del Valle del Mantaro. Los turistas al llegar a los lugares visitados, demandan alimentos, bebidas, productos artesanales, y compran de las familias que expenden y de este modo el turismo genera fuente de trabajo y de ingreso en beneficio de los lugareños.

Asimismo, en todos los distritos del Valle del Mantaro, de norte a sur y viceversa todos los días de la semana se realizan las famosas ferias rurales y funcionan de lunes a domingo, son verdaderos circuitos comerciales. La feria de Huayucachi, en el distrito del mismo nombre, funciona en una infraestructura adecuada y bien organizada, lugar donde los campesinos de la zona sur del Valle del Mantaro aprovechan para vender sus productos agropecuarios, artesanales, flores, frutas, lana, animales mayores y menores, granos y con el dinero de la venta, compran productos de origen industrial como los alimentos, ropas. Para los comerciantes son espacios de empleo y de ingreso rodante. Los comerciantes ya tienen puestos para realizar las transacciones comerciales. Las ferias rurales vienen a constituir el verdadero circuito comercial, permite la interrelación permanente entre el campo y la ciudad y viceversa (campo.-.ciudad, rural.-.urbano) y forma parte de una unidad espacial articulado y en permanente interacción socioeconómico y cultural.

\section{MARCO TEÓRICO}

Mercado rural es un concepto que hace referencia al espacio en el cual funcionan mercados de bienes y factores particulares cuya base territorial y social es el campo. Sin embargo, la existencia del campo es imprecisa y sólo se entiende en su relación con la ciudad. (En realidad, es preferible referirse al binomio mercado ruralmercado urbano como una unidad espacial: el mercado regional.) En nuestro concepto (Gonzáles 1982, 1984, 1987, 1994), el mercado regional es la unidad de análisis espacial apropiada para entender sus componentes. Será desde esta perspectiva que haremos el balance de la investigación sobre el tema. De acuerdo con ella, se incorporan en el análisis sectores productivos como la minería, la pesquería, la explotación forestal, la pequeña industria, el comercio, el transporte y los servicios rurales, 
aparte de los clásicos, los sectores agrícolas y pecuario (Olart, 1994:23)

Del mismo modo cabe mencionar a los autores: los de Thorp \& Bertram (1978), Hopkins (1981) y Caballero (1981) son de los pocos estudios globales que, a escala nacional, incluyen análisis sobre los mercados rurales de productos y factores. Los primeros subrayan la importancia de la articulación de productores agropecuarios capitalistas y no capitalistas al mercado mundial a través de la exportación de azúcar, algodón, café y lanas, lo que ha repercutido en el largo plazo sobre la estructura agraria y ha definido los términos de la participación de los distintos segmentos de agricultores en la economía de mercado. Precisamente Hopkins (1981) y Álvarez (1983) contribuyeron con una clasificación de los productores agropecuarios en función de su participación en cuatro tipos de mercado distintos: mercados de exportación, urbanos, agroindustriales y mercados restringidos, es decir, aquellos mercados rurales con fuerte participación campesina.

Esta tipología ha sido bastante utilizada posteriormente, tanto en las investigaciones como en trabajos orientados hacia las políticas de desarrollo. Asimismo, Caballero, en 1981, contribuyó con un gran fresco sobre la región neurálgica del Perú: la sierra, señalando que el desarrollo del mercado hizo parte de la "destrucción capitalista» de la vieja economía y sociedad serranas, y la "construcción capitalista» trajo consigo nuevas formas de mercado, sobre todo el de trabajo. De esta manera se generó un proceso de cambio denominado "la gran transformación", cuya heterogeneidad se habría debido al origen exógeno del desarrollo capitalista y a las escasas y desiguales condiciones que ofrece la sierra para dicho desarrollo.

El Perú no tenía -y aún no tiene- un mercado nacional integrado, debido a la desigualdad del desarrollo capitalista por regiones. Éstas, siendo unidades ciudad-campo, no habían podido constituir un sistema de mercados con iguales grados de desarrollo; en consecuencia, había diferencia sustantiva entre los cuatro tipos de ciudades que definimos en aquel entonces (Gonzáles 1982).

(Hopkins 1981), los mercados microrregionales (Gonzáles 1982, 1987, 1994) o las microrre- giones (Plaza y Francke 1981), es decir, aquellos pequeños valles, cuencas o altiplanos donde los mercados funcionaban temporalmente, no incorporaban toda la producción y coexistían con economías de autosubsistencia, y donde, en consecuencia, existían y aún existen instituciones alternativas de asignación de recursos como son la comunidad campesina, las juntas de regantes o de pastoreo (Olarte).

La importancia de la región para explicar el funcionamiento de los mercados ha sido objeto de atención menos genérica y más minuciosa en los estudios de los geógrafos, los historiadores y los sociólogos.

Appleby (1976), Brisseau (1975) y Watters (1994) son tres geógrafos que investigaron sobre tres regiones, analizando los mercados existentes dentro de sus respectivos paisajes geográficos.

Claverías (1978) se ocupa de las transformaciones de las relaciones de producción por el desarrollo del mercado interno y el cambio de la lógica de explotación terrateniente, y su repercusión sobre los movimientos campesinos en Puno en la década de 1950. El principal mecanismo de desarrollo del mercado interno fue el incremento de la diferenciación campesina, es decir, la mayor proletarización de los campesinos con escaso ganado o tierra, debido al aumento del «yerbaje» o derecho de pastoreo cobrado por los hacendados. Sin embargo, alcanzó sus límites cuando los movimientos campesinos organizados espontáneamente impidieron el desalojo de sus tierras y, con ello, frenaron el incremento de la proletarización y el desarrollo del mercado de fuerza de trabajo.

El historiador Nelson Manrique (1987) es uno de los pocos que explicita la definición de mercado interno como: «... por una parte, un proceso nacional; por otra, supone no solamente la mercantilización general de la economía sino, fundamentalmente, la conversión de la propia fuerza de trabajo en una mercancía: la creación de un mercado de trabajo asalariado a través de la proletarización de una fracción de los productores pre capitalistas» (p. 265).

El estudio que más se aproxima al análisis de Páginas 52-58 
mercados de productos es el de Scott (1985) sobre la comercialización de la papa en la zona central del Perú. Se trata de un minucioso análisis de las etapas que recorre la papa para llegar de la chacra a la olla y de los distintos actores e instituciones que intervienen en este mercado. Allí se analiza el problema de la gran brecha existente entre el precio en chacra y el precio al consumidor final. El principal aporte del libro es que la extensa cadena de comercialización 110 sólo activa mercados microrregionales y regionales del producto, sino que también activa el mercado de trabajo para rescatistas, transportistas, cargadores, almaceneros, mayoristas, repartidores y minoristas, cuyos ingresos explican el encarecimiento de la papa. Es decir, los mercados de productos son algo más que la simple compra-venta del producto mismo. Faltan análisis como el de Scott y de los participantes en el libro de Escobal (1994) para entender mejor los mercados rurales por productos.

Mercados rurales y su articulación.- La articulación de mercados entendida como la interrelación entre los mercados de factores y de éstos con los mercados de bienes rurales y urbanos, es un fenómeno poco o nada estudiado. Los trabajos de Fuenzalida y otros (1982) sobre la articulación de los campesinos de Huayopampa en el mercado de Lima, Figueroa y Ruiz (1984) y Ruiz (1980) sobre términos de intercambio campo-ciudad y Gonzales (1987) sobre inflación rural, son escasos ejemplos que tratan de manera indirecta el problema de la articulación de mercados rurales y urbanos. El tema es muy importante para las políticas económicas y para el ajuste estructural, pues si no se conoce cómo influye un mercado sobre el desempeño de otro, las políticas de corto y largo plazo se diseñan y realizan a tientas (Olarte).

\section{MATERIAL Y MÈTODOS \\ Materiales y equipos}

En el proceso de la investigación se utilizaron fichas de entrevista como materiales, los mismo fueron aplicados a los directivos que administran los circuitos turísticos del Valle del Mantaro, a los ofertantes de la ferias y de los circuitos, se utilizaron la cámara fotográfica, filmadora, altímetro, GPS, para determinar los puntos, los

Páginas 52-58 cuestionarios de preguntas para aplicar a los beneficiarios de los circuitos turísticos y de este modo obtener los datos de ingresos y opiniones de los beneficios que brindan servicios a los turistas en los distrito involucrados en este tipo de actividades-

\section{Métodos}

Los método de investigación fueron el análisis y síntesis, el inductivo y deductivo. Los datos fueron obtenidos mediante la aplicación de la técnica de los cuestionarios, entrevistas a los pequeños comerciantes en las distintas ferias y el universo de la población y/o muestra formaron parte a los feriantes y los ofertantes a los turistas en los distritos involucrados a esta actividad. Los instrumentos utilizados fueron la ficha de entrevista y en el procesamiento de los datos se utilizó Minitab y en el análisis participó el estadístico Mauro Rafaele de la Cruz, principalmente en el procesamiento de los datos y en la prueba de hipótesis.

\section{RESULTADOS.}

Los circuitos turísticos y las ferias rurales han incrementado el nivel de ingreso económico de las familias beneficiarias del Valle del Mantaro y del mismo modo han mejorado significativamente en el nivel educativo, salud y en sus condiciones de vida, han mejorado las vivienda, les ha permitido invertir en la compra de vehículos y dotarse de infraestructura turística para ofertar en mejores condiciones a los visitantes. 
CUADRO DE FERIAS RURALES EN EL VALLE DEL MANTARO Y PUEBLOS VECINOS

INFLUENCIA DE LAS FERIAS

\begin{tabular}{|c|c|c|c|}
\hline DÍAS & LUGAR & $\begin{array}{l}\text { DISTANCIA DE } \\
\text { HUANCAYO }\end{array}$ & CARACTERÍSTICAS \\
\hline LUNES & Huayucachi & $8,7 \mathrm{~km}$ sur de Huancayo & Agropecuaria \\
\hline MARTES & $\begin{array}{l}\text { Viques } \\
\text { Pucará, } \\
\text { San Agustín de Cajas }\end{array}$ & $\begin{array}{l}12 \mathrm{Km} \text { sur de Hyo } \\
15 \mathrm{Km} \text { sur de Hyo } \\
8 \mathrm{~km} \text {. Norte Hyo. }\end{array}$ & $\begin{array}{l}\text { Agropecuario } \\
\text { Agropecuario } \\
\text { Agropecuarios }\end{array}$ \\
\hline \multirow[t]{3}{*}{ MIÉRCOLES } & $\begin{array}{l}\text { San Jerónimo de Tunan, } \\
\text { Hualhuas } \\
\text { Jauja }\end{array}$ & $\begin{array}{l}16 \mathrm{~km} \text { norte Hyo } \\
10 \mathrm{Km} \text { norte de Hyo } \\
45 \mathrm{Km} \text {. Norte de hyo }\end{array}$ & $\begin{array}{l}\text { Agropecuario } \\
\text { Agropecuario y artesanal }\end{array}$ \\
\hline & & & Agropecuario, artesanal e \\
\hline & & & industrial \\
\hline JUEVES & $\begin{array}{l}\text { Orcotuna } \\
\text { Sicaya } \\
\text { Huancán } \\
\text { Sapallanga } \\
\text { Concepción }\end{array}$ & $\begin{array}{l}16 \text { km norte de Hyo. } \\
10 \mathrm{~km} \text {. oeste de Hyo. } \\
5 \mathrm{~km} \text { sur Hyo. } \\
8 \mathrm{~km} \text { sur Hyo. } \\
20 \mathrm{Km} \text { norte de Hyo }\end{array}$ & $\begin{array}{l}\text { Agropecuario } \\
\text { Agropecuario } \\
\text { Agropecuario } \\
\text { Agropecuario } \\
\text { Agropecuario }\end{array}$ \\
\hline VIERNES & $\begin{array}{l}\text { Ahuac } \\
\text { Aco } \\
\text { Chongos Bajo } \\
\text { Pazos } \\
\text { Acostambo } \\
\text { Chaquicocha } \\
\text { Cochas Chico }\end{array}$ & $\begin{array}{l}15,6 \mathrm{~km} \text {. Oeste Hyo } \\
16 \mathrm{Km} \text { norte Hyo. } \\
17,2 \mathrm{~km} \text { oeste de Hyo. } \\
47 \mathrm{Km} \text { de sur de Hyo. } \\
50 \mathrm{Km} \text { sur de Hyo. } \\
48 \mathrm{Km} \text {. Oeste de Hyo. } \\
11 \mathrm{~km} \text { este Hyo. }\end{array}$ & $\begin{array}{l}\text { Agropecuario } \\
\text { Agropecuario } \\
\text { Agropecuario } \\
\text { Agropecuario } \\
\text { Agropecuario } \\
\text { Agropecuario } \\
\text { Agropecuario }\end{array}$ \\
\hline SÁBADO & $\begin{array}{l}\text { Chupaca, } \\
\text { Marco } \\
\text { Matahasi } \\
\text { Muquiyauyo }\end{array}$ & $\begin{array}{l}\text { 8,8 km oeste de Hyo. } \\
60 \mathrm{Km} \text { norte de Hyo. } \\
25 \mathrm{Km} \text { norte de Hyo. } \\
30 \mathrm{Km} \text {. Norte de Hyo. }\end{array}$ & $\begin{array}{l}\text { Agropecuario } \\
\text { Agropecuario } \\
\text { Agropecuario } \\
\text { Agropecuario }\end{array}$ \\
\hline DOMINGO & $\begin{array}{l}\text { Huancayo, } \\
\text { Concepción } \\
\text { Jauja } \\
\text { Mito } \\
\text { Coto coto }\end{array}$ & $\begin{array}{l}21 \mathrm{~km} \text { norte de Hyo. } \\
47 \mathrm{~km} \text { norte Hyo. } \\
31 \mathrm{~km} \text { margen derecha } \\
5 \mathrm{Km} \text { de sur de Hyo. }\end{array}$ & $\begin{array}{l}\text { Agropecuario } \\
\text { Agropecuario y artes. } \\
\text { Agropecuario } \\
\text { Agropecuario } \\
\text { Agropecuario }\end{array}$ \\
\hline TOTAL & 27 & & \\
\hline
\end{tabular}




\section{Ferias rurales y circuitos turísticos en el ingreso económico de las familias ofertantes.}

Tabla 1. Productos turísticos que ofertan los ofertantes

\begin{tabular}{lccc}
\hline $\begin{array}{c}\text { Producto tu- } \\
\text { rístico }\end{array}$ & $\begin{array}{c}\text { Ferias } \\
\text { rurales }\end{array}$ & $\begin{array}{c}\text { Circuitos } \\
\text { turísticos }\end{array}$ & Total \\
\hline $\begin{array}{l}\text { Agropecua- } \\
\text { rio }\end{array}$ & 44,0 & & 30,9 \\
$\begin{array}{l}\text { Gastrono- } \\
\text { mía }\end{array}$ & 54,1 & 32,7 & 47,7 \\
$\begin{array}{l}\text { Amenida- } \\
\text { des }\end{array}$ & & 67,3 & 20,1 \\
Artesanías & 1,9 & & 1,4 \\
\hline \multicolumn{1}{c}{ Total } & $\begin{array}{l}100,0 \\
(259)\end{array}$ & $\begin{array}{l}100,0 \\
(110)\end{array}$ & $\begin{array}{c}100,0 \\
(369)\end{array}$ \\
\hline
\end{tabular}

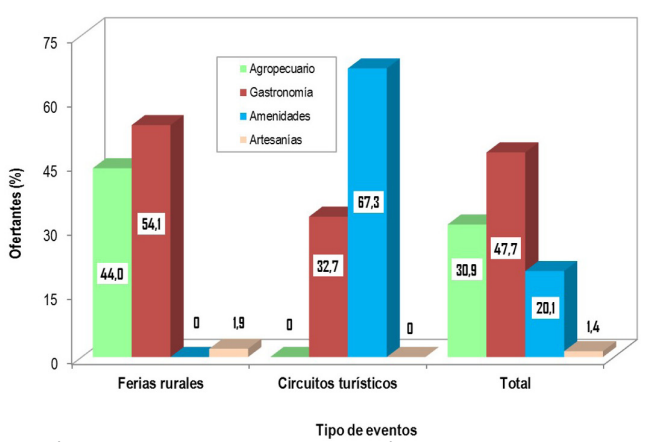

Figura 1. Producto turístico que ofertan los afertantes

En la tabla 1 y figura 1 se constata que el 47,7\% de los ofertantes del Valle del Mantaro ofertan productos de gastronomía, el $54,1 \%$ de los ofertantes de ferias rurales y el $32,7 \%$ de los ofertantes de circuitos turísticos. La mayoría de los ofertantes de los circuitos turísticos ofrecen amenidades. El 30,9\% de los ofertantes ofrecen productos agropecuario, el 20,1\% amenidades y el 1,4\% artesanías.

En la tabla 7 y gráfico 6 se descubre que el $43,1 \%$ de los ofertantes del Valle del Mantaro se dedican a la actividad ferial o turística de 20 años a más, el $24,4 \%$ menos de 5 años, el $20,3 \%$ de 10 a 19 años y el $12,2 \%$ de 5 a 9 años. La mayoría de los ofertantes de ferias rurales $(59,5 \%)$ se dedican de 20 años a más y la mayoría de los ofertantes de los circuitos turísti$\cos (63,6 \%)$ por menos de 5 años.

Tabla 2. Ingreso mensual por la actividad ferial y turística de los ofertantes

\begin{tabular}{cccc}
\hline $\begin{array}{c}\text { Ingresos } \\
(\mathrm{S} / .)\end{array}$ & Ferias rurales & $\begin{array}{c}\text { Circuitos } \\
\text { turísticos }\end{array}$ & Total \\
\hline$<300$ & 49,8 & 18,2 & 40,4 \\
\hline 300 a 500 & 23,2 & 43,6 & 29,3 \\
\hline $\begin{array}{l}501 \mathrm{a} \\
1500\end{array}$ & 11,6 & 22,7 & 14,9 \\
\hline$>1500$ & 15,4 & 15,5 & 15,4 \\
\hline Total & $\begin{array}{l}100,0 \\
(259)\end{array}$ & $\begin{array}{l}100,0 \\
(110)\end{array}$ & $\begin{array}{c}100,0 \\
(369)\end{array}$ \\
\hline
\end{tabular}

En la tabla 2 y figura 2 se descubre que el $40,4 \%$ de los ofertantes del Valle del Mantaro perciben ingresos económicos mensuales por debajo de los 300 soles, el $49,8 \%$ de los ofertantes de ferias rurales y el $18,2 \%$ de los ofertantes de circuitos turísticos. El 29,3\% de los ofertantes perciben de 300 a 500 soles, el $14,9 \%$ de 501 a 1500 soles y el $15,4 \%$ más de 1500 soles. El $43,6 \%$ de los ofertantes de circuitos turísticos perciben de 300 a 500 soles.

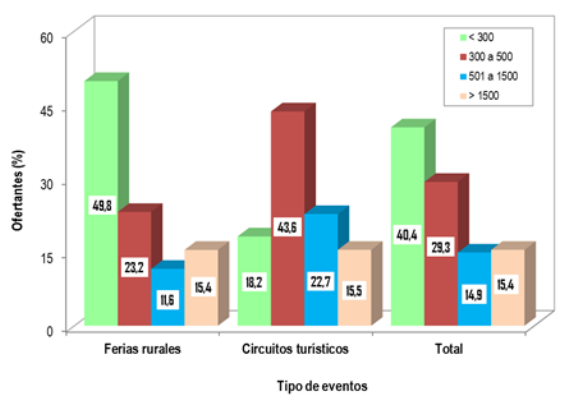

Figura 2. Ingreso mensual por la actividad ferial y turistica de los ofertantes 


\section{DISCUSIÓN.}

Es evidente que las ferias rurales y los circuitos turísticos vienen generando fuente de empleo y de ingreso no solamente para un miembro de la familias, sino para todos los miembros y por tanto significa ocupación familiar, organizados en empresas familiares, los mismos permite incrementar el nivel de ingreso económico, lo cual genera accesibilidad a la educación, salud, vivienda y gozar mejores condiciones de vida para el bienestar de las familias rurales y urbanas que están involucradas en estas actividades ya mencionadas. El resultado de trabajos similares consideran que el turismo es un factor de de ingreso y empleo al igual que las ferias rurales existes en el ámbito del Valle del Mantaro.

El Estudio se llegó a las siguientes conclusiones:

1. Los circuitos turísticos y las ferias rurales han incrementado el nivel de ingreso económico de las familias beneficiarias del Valle del Mantaro. 2. Los circuitos turísticos y las ferias rurales han mejorado el nivel educativo, de salud y las condiciones de la vivienda de las familias beneficiarias del Valle del Mantaro.

3. Los circuitos turísticos y las ferias rurales vienen modificando los patrones culturales de las familias beneficiarias del Valle del Mantaro.

\section{AGRADECIMIENTOS}

A los y las comerciantes de las ferias de Huayucachi, Chaquicocha, Concepción, Jauja, feria dominical de Huancayo, de Coto coto, por haber proporcionado información de manera incondicional.

\section{REFERENCIAS}

Ágreda, Víctor (1992). El mercado internacional y los productores de economía campesina y agricultura comercial en Perú: El problema agrario en debate. SEPIA IV. Lima: SEPIA, pp. 103-134.

Alberti, Giorgio y Enrique Mayer, compiladores (1974). Reciprocidad e intercambio en los Andes peruanos. Lima: IEP. Serie Perú Problema $\mathrm{N}^{\circ} 12$.

Alvarado, Javier (1985). Mercado de crédito campesino: El caso de la comunidad de Aricato. Lima: Pontificia Universidad Católica del Perú. Tesis de Maestría,
Alvarado, Javier (1997). En los límites de la economía de mercado: El crédito campesino. En E. Gonzales y otros: La lenta modernización de la economía campesina. Lima: IEP. Serie Análisis Económico $N^{\circ} 12$.

Alvarado, Javier (1990.) Mercados financieros rurales: Posibilidades y límites. En Perú: El problema agrario en debate. SEPIA III. Lima: SEPIA, pp. 291-304.

Alvarado, Javier (1994). La naturaleza de las transacciones de crédito en el medio rural. En Perú: El problema agrario en debate/SEPIA V. Lima: SEPIA. UNSA. CAPRODA, pp. 457-475,

Gonzales de Olarte, Efraín (2009). Mercados en el ámbito rural peruano, editorial SEPIA, Lima. 